\title{
Deeply virtual Compton scattering
}

\section{Patrick R.B. Saul* on behalf of the $\mathbf{H 1}$ and ZEUS collaborations}

Deutsches Elektronen-Synchrotron DESY, Notkestrasse 85, 22603 Hamburg, Germany E-mail: patrick. sauli@desy.dei

ABstract: Recent HERA results from H1 and ZEUS on deeply virtual Compton scattering (DVCS), $e^{+} p \rightarrow e^{+} \gamma p$, are presented. The DVCS process is measured as an excess of photons in deep inelastic scattering data above the QED Compton expectation in the central region of the detectors. Differential cross sections as a function of photon virtuality, $Q^{2}$, and $\gamma^{*} p$ centre-of-mass energy, $W$, are presented for $Q^{2}>2 \mathrm{GeV}^{2}$ and $30<W<140 \mathrm{GeV}$. The data are in broad agreement with QCD model predictions.

\section{Introduction}

Deeply virtual Compton scattering (DVCS) [i] in is the diffractive production of a photon in deep inelastic ep scattering, Fig. $i_{-i}^{-}$It interferes with the well-known QED Compton

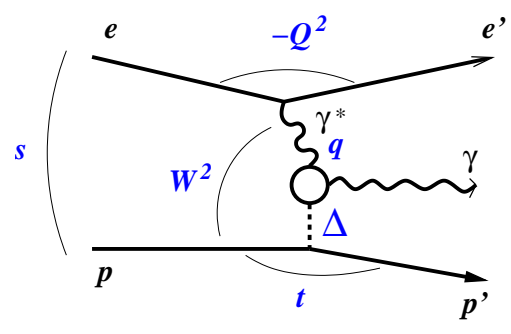

Figure 1: Diagram for the DVCS process.
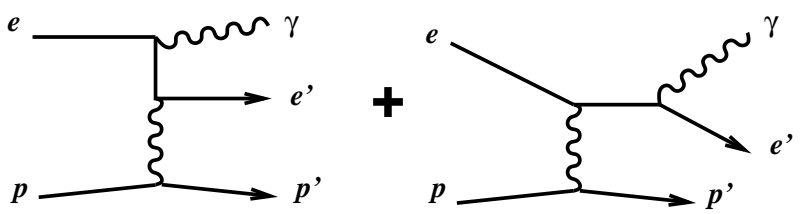

Figure 2: Diagrams for the QEDC process.

(QEDC), or Bethe-Heitler (BH), process shown in Fig. $\overline{2}_{r}^{2}$ Through its relation to generalised parton distributions, the DVCS process is of great theoretical interest [2i]. QCDbased model predictions for DVCS from Frankfurt, Freund, and Strikman (FFS) [3in] and Donnachie and Dosch (DD) [4] This allows a confrontation of the theoretical understanding of DVCS with measured data. Note, however, that theory is thus far unable to predict the dependence on $t$, the fourmomentum transfer squared at the proton vertex, although it is generally assumed to be exponential, $e^{b t}$, with $b$ expected to lie in the range 4 to $10 \mathrm{GeV}^{-2}$ for HERA data.

${ }^{*}$ Speaker. 
Investigating DVCS at HERA is challenging. Its total cross section is much smaller than that of the QEDC process. The scattered proton is not observed and the positron typically scatters outside the tracking acceptance, so candidate events usually have no vertex and the kinematics are poorly reconstructed. Nevertheless, the first observation was reported by the ZEUS Collaboration in 1999 [9.9. Since then both the H1 and ZEUS Collaborations have produced DVCS cross-section measurements [i] between the H1 and ZEUS analyses is given in Table Is. Essentially, ZEUS uses more luminosity and $\mathrm{H} 1$ extends the measurements to lower $Q^{2}$.

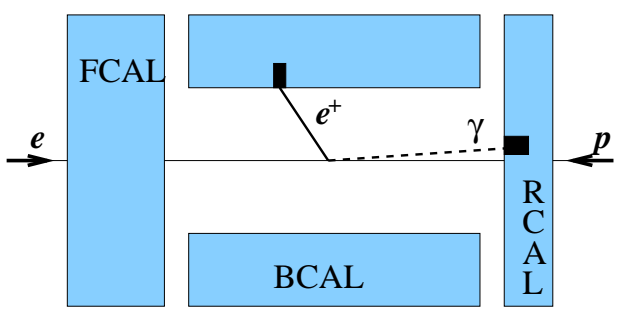

Figure 3: Control sample toplogy.

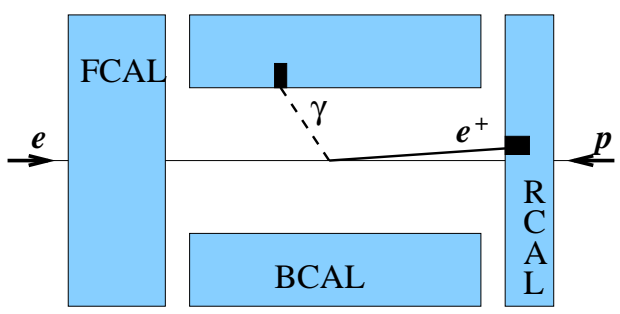

Figure 4: Enriched sample topology.

The strategy for extracting a DVCS cross section is similar for both H1 and ZEUS. Candidate $e^{+} p \rightarrow e^{+} \gamma p$ events are selected with 2 electromagnetic (EM) clusters in the detector, one in the central region and one in the rear, defined by the positron beam direction. These are then subdivided into 2 mutually exclusive samples. The control sample (Fig. $\left.\underline{3}_{-1}\right)$ contains events in which the EM candidate in the central region has an associated positively-charged track. This sample is dominated by QEDC events. The enriched DVCS sample (Fig. 䢙) contains events in which the central-region EM candidate has no associated track. This sample is contains mainly DVCS and QEDC events. If the QEDC prediction provides a good description of the control sample, then it can be used to subtract this contribution from the enriched DVCS sample, thus yielding a measurement of the DVCS cross section. Note that a direct subtraction of the QEDC contribution is a

\begin{tabular}{|l|l|}
\hline H1 & ZEUS \\
\hline \multicolumn{2}{|c|}{ Main selection requirements: } \\
\hline 1 central EM cluster with $p_{T}>1 \mathrm{GeV}$ & 1 central EM cluster with $E_{T}>3 \mathrm{GeV}$ \\
\hline 1 rear EM cluster with $E>15 \mathrm{GeV}$ & 1 rear EM cluster with $E>10 \mathrm{GeV}$ \\
\hline $2<Q^{2}<20 \mathrm{GeV}^{2}$ & $5<Q^{2}<100 \mathrm{GeV}^{2}$ \\
\hline $30<W<120 \mathrm{GeV}$ & $40<W<140 \mathrm{GeV}$ \\
\hline$-1.15<\eta_{\text {central cluster }}<1.5$ & $-0.6<\eta_{\text {central cluster }}<1.0$ \\
\hline \multicolumn{2}{|c|}{ Events satisfying selection cuts: } \\
\hline $338(e)+172(\gamma)$ from $8 \mathrm{pb}^{-1}$ Cross section definitions: \\
\hline \multicolumn{2}{|c|}{$1059(e)+537(\gamma)$ from $37 \mathrm{pb}^{-1}$} \\
\hline $2<Q^{2}<20 \mathrm{GeV}^{2}, 30<W<120 \mathrm{GeV}$ & $5<Q^{2}<100 \mathrm{GeV}^{2}, 40<W<140 \mathrm{GeV}$ \\
\hline$|t|<1 \mathrm{GeV}^{2}$ & $E_{T}^{\gamma}>3 \mathrm{GeV}^{2}-0.6<\eta_{\gamma}<1.0$ \\
\hline
\end{tabular}

Table 1: Comparison of H1 and ZEUS analyses. 
valid procedure because in the measured kinematic region the interference term between the two 1) is predicted to be small, and 2) is approximately proportional to the cosine of the difference in the azimuthal scattering angles of the positron and proton in the $\gamma^{*} p$ frame, which integrates to zero since the proton is not observed.

\section{H1 Results}

Plots of kinematic variables for the H1 analysis are shown in Fig. $\underset{\text { s. }}{\mathbf{p}}$. On the left, the uncorrected kinematic distributions are shown for the control sample. The data are compared with predictions for the $\mathrm{BH}$ contribution, and for small di-lepton and diffractive $\rho$ production background contributions. The sum of these contributions provides a good description of the data, thus providing confidence that the detector response and background contributions are well-understood. On the right in Fig. '. the same kinematic distributions are shown for the enriched DVCS sample. There is a clear excess above the $\mathrm{BH}$ and diffractive $\omega$ and $\phi$ contributions which cannot be explained by a change in the overall normalisation of the $\mathrm{BH}$ contribution.
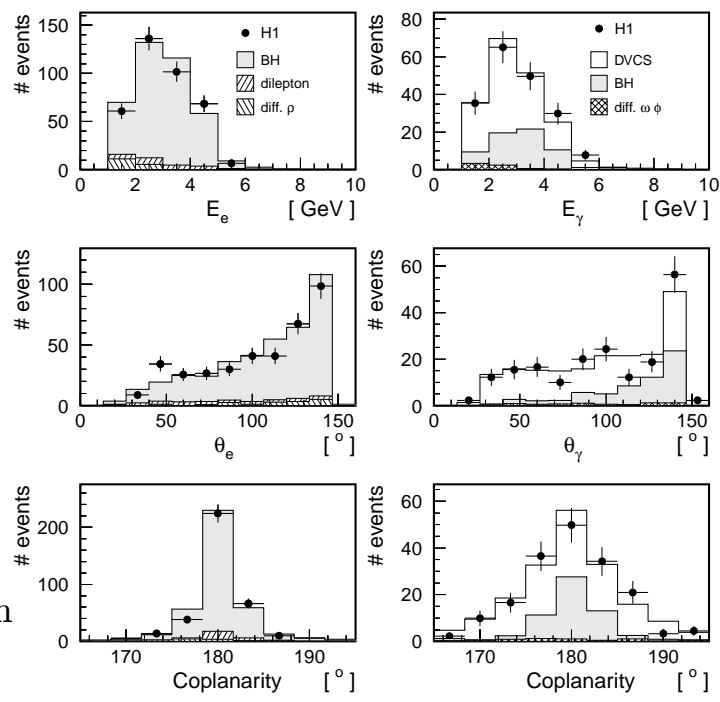

Figure 5: Event distributions of the control sample (left) and of the enriched DVCS sample (right).

However, the introduction of the DVCS process, here scaled to agree with the total number of events in the data, is able to account for the excess very well.

In Figs. $\overline{6}_{1}^{\prime}$ and $\overline{7}_{1}$, the $\mathrm{BH}$ and background contributions have been subtracted and

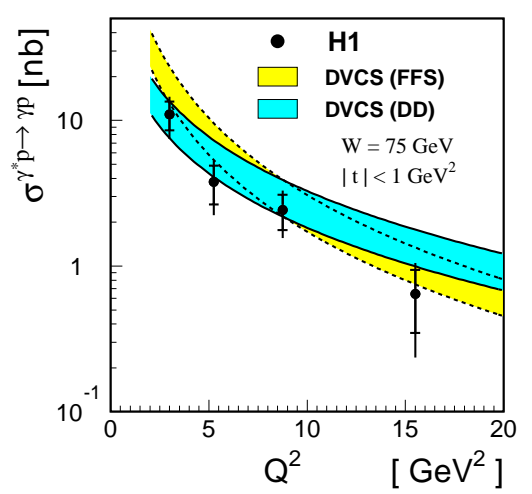

Figure 6: DVCS cross section for the process $\gamma^{*} p \rightarrow \gamma p$ as a function of $Q^{2}$.

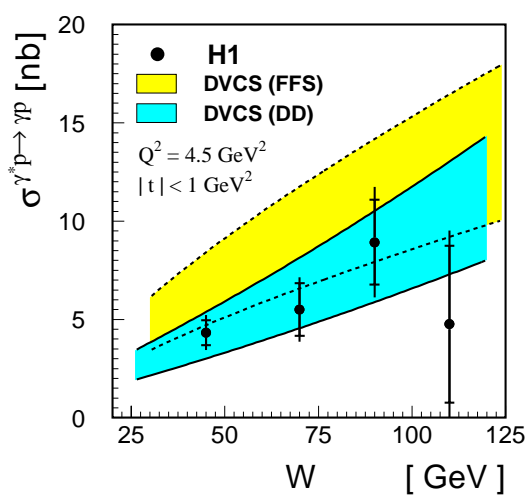

Figure 7: DVCS cross section for the process $\gamma^{*} p \rightarrow \gamma p$ as a function of $W$. 
the data presented as a $\gamma^{*} p \rightarrow \gamma p$ DVCS cross section. There is broad agreement with the overlaid predictions by FFS and DD. The shaded bands represent the change in the overall normalisation of the theory introduced by a variation in the $t$-slope $b$ in the range 5 to $9 \mathrm{GeV}^{-2}$, thus demonstrating the need for a better prediction of $b$ and/or a direct measurement of the $t$ distribution using data.

\section{ZEUS Results}

In Fig. 18i, kinematic distributions are shown for the ZEUS control sample. The data are

ZEUS

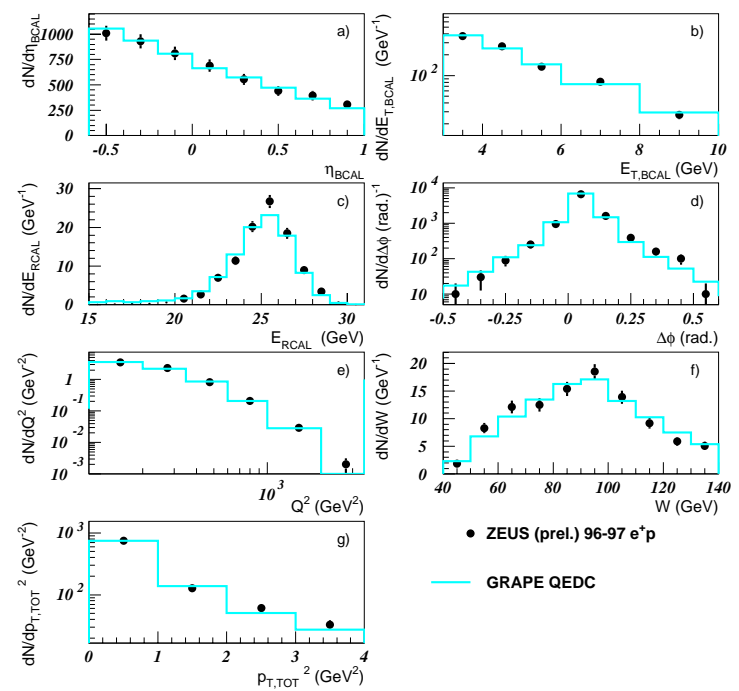

Figure 8: Event distributions for the control sample.
ZEUS

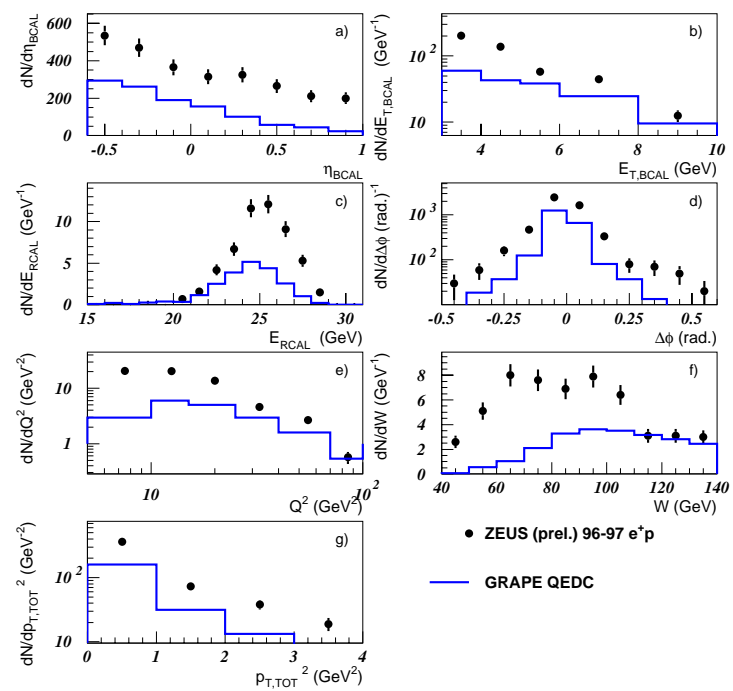

Figure 9: Event distributions for the enriched DVCS sample.

compared with the QEDC prediction, normalised to the number of events in the data. A very good description is obtained. Note that in contrast to the $\mathrm{H} 1$ analysis, at higher $Q^{2}$ no simulation of additional vector meson backgrounds is required, although a small (3\%) contribution from di-lepton production (not shown) has been subtracted.

In Fig. $\bar{p}_{1}^{\prime}$, the same kinematic distributions are shown for the enriched DVCS sample. The overlaid QEDC prediction, normalised according to the control sample, is unable to account for the data in either shape or normalisation. After subtracting this contribution, the data are in good agreement with the FFS prediction. The resulting DVCS cross sections

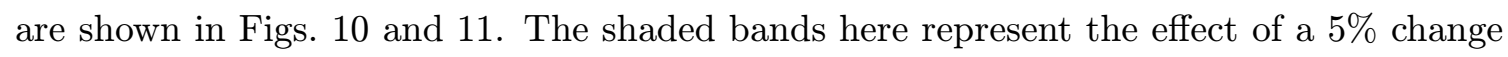
in the energy scale of the calorimeter. Noting that about a $20 \%$ contribution from protondissociation has not been subtracted, the overlaid prediction by FFS reproduces the data in both shape and normalisation using a $t$-slope value of $b=4.5 \mathrm{GeV}^{-2}$.

\section{Summary and Outlook}

An excess of $e p \rightarrow e p \gamma$ events over that expected from the QEDC process has been clearly established by both the $\mathrm{H} 1$ and ZEUS Collaborations. The kinematic distributions of this 


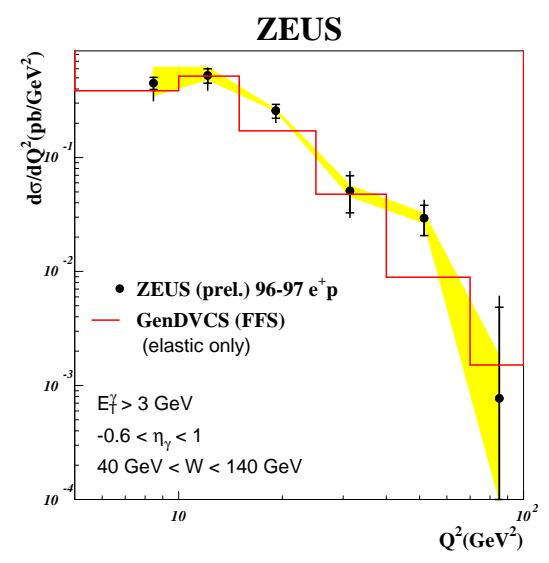

Figure 10: DVCS cross section for the process $e p \rightarrow e p \gamma$ as a function of $Q^{2}$.

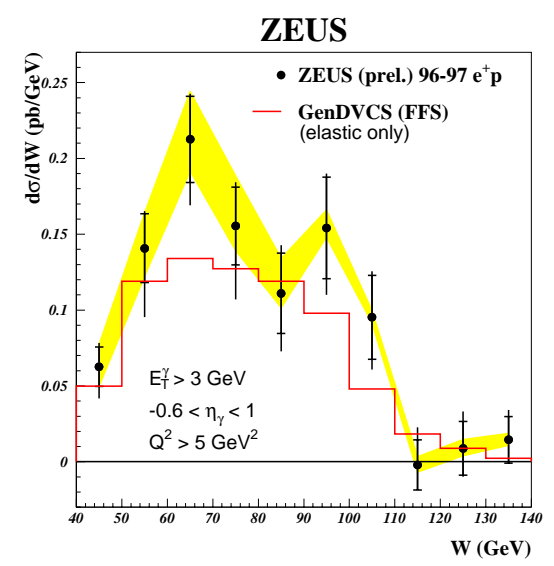

Figure 11: DVCS cross section for the process $e p \rightarrow e p \gamma$ as a function of $W$.

excess are inconsistent with those of QEDC scattering. QEDC and other backgrounds have been subtracted and DVCS cross sections have been measured. QCD-based model predictions are generally able to reproduce these measurements, although a large theoretical uncertainty in the normalisation exists owing to the lack of knowledge of the $t$-slope value.

DVCS analysis at HERA is only just beginning. Larger datasets exist on tape from the '98-'00 running periods, and HERA promises another approximately $1 \mathrm{fb}^{-1}$ per experiment in the next five years, including longitudinal polarisation of the lepton beam. Future measurements will also focus on the $t$-distribution, the interference term, and ultimately skewed parton distributions.

\section{References}

[1] E. Wieczorek, V.A. Matveev and D. Robaschik, Theor. Math Phys. 19

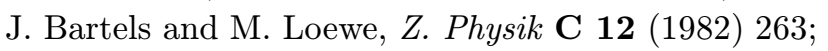

X. Ji, 'Phys. Rev. D 55 (1997) 7114 .

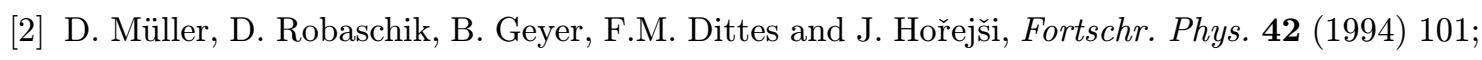
X. Ji, $\bar{P} \bar{h} y$ s. Rev. Lett. $\overline{7} \overline{8}(19 \overline{9} \overline{7}) 610$

A.V. Radyushkin, Phys.Rev. D $\mathbf{5 6}(1997) 5524$.

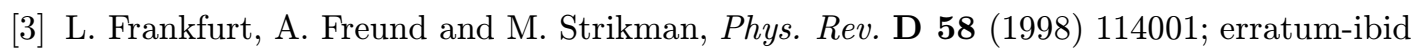

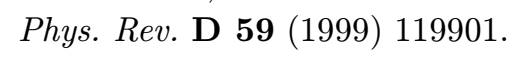

[4] A. Donnachie and H.G. Dosch, Phys. Lett. B 502 (2001) 74t.

[5] P.R.B. Saull, A Monte Carlo Generator for Deeply Virtual Compton Scattering at HERA, 1999, available at http://www-zeus.desy.de/ saull/public/dvcs.html.

[6] R.Stamen, Ph.D. dissertation, Universität Dortmund, November 2001.

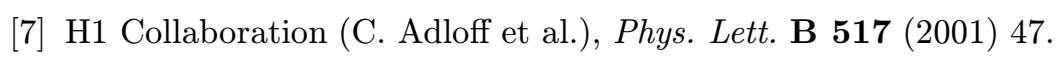

[8] ZEUS Collaboration, Abstract 564, paper submitted to EPS Conf. on HEP, Budapest 2001.

[9] ZEUS Collaboration (P.R.B. Saull for the collaboration), Prompt photon production and observation of Deeply Virtual Compton scattering, published in the EPS-HEP99 proceedings, Tampere, Finland, 15-21 July, 1999 [hep-ex/0003030i]. 